\title{
CONDIÇÕES MAIS FREQUENTES EM UM AMBULATÓRIO DE PERÍCIA NEUROLÓGICA
}

\author{
Paulo Cesar Trevisol-Bittencourt ${ }^{1}$, Michelangelo Antunes Ferreira ${ }^{2}$, \\ Antônio Carlos Marasciulo ${ }^{3}$, Carlos Fernando Collares ${ }^{4}$
}

\begin{abstract}
RESUMO - Objetivo: Apresentar as condições nosológicas vistas em perícia neurológica em um ambulatório de referência estadual do Instituto Nacional de Seguridade Social (INSS), Florianópolis, Santa Catarina, Brasil, durante o período de outubro de 97 a maio de 98. Método: Revisão dos relatórios especializados das perícias solicitadas ao especialista em neurologia, com identificação dos diagnósticos finais dos 108 pacientes encaminhados para avaliação. Resultados: Os diagnósticos mais comuns em ordem decrescente de frequência foram: epilepsia, doenças reumatológicas, distúrbios psiquiátricos, alterações neurológicas associadas a alcoolismo crônico, síndrome pós TCE e doença vascular cerebral. Conclusões: Doenças neurológicas podem determinar importante grau de incapacidade no trabalhador catarinense. Contudo, existe um subestimado potencial de recuperação funcional. Além disso, diversas condições não genuinamente neurológicas são encaminhadas para avaliação especializada.
\end{abstract}

PALAVRAS-CHAVE: perícia médica, neuroepidemiologia, epilepsia.

The most common disorders in neurological clinic for medico-legal assessment of labour capacity at the Brazilian Social Security System (INSS) in Florianópolis - SC, Brazil.

\begin{abstract}
Objective: To present the most frequent diagnosis among patients refered for neurological evaluation to estimate their labour capacities at the unit of National Institute of Social Security (INSS), Florianópolis-SC, southern Brazil. Method: Review of all medical records of 108 patients evaluated between October 97 and May 98. The sample was submited to judicious medico-legal assessment to define their final diagnosis. Results: Neurological evaluation disclosed as the commonest disorders, in decreasing order of frequency: epilepsy, rheumatic diseases, psychiatric illnesses, neurological disorders related to chronic alcoholism, head trauma syndrome and cerebrovascular diseases. Conclusion: Neurological disorders may be responsible for important disability among workers in our society. However, the potential for social rehabilitation, often underestimated, must be considered. Moreover, diverse non-neurological conditions used to be sent for neurological evaluation.
\end{abstract}

KEY WORDS: medico-legal report, neuroepidemiology, epilepsy.

Quais as características de uma população avaliada por incapacidade laborativa em um ambulatório de neurologia no Brasil? Na literatura médica atual não existem respostas ou dados claros para esta questão. Nações desenvolvidas têm por praxe investigar quais as condições nosológicas mais frequentemente associadas com incapacidade laboral ${ }^{1}$. Aliás, este conhecimento é um requisito essencial para o desenvolvimento de estratégias visando a prevenção e controle das mesmas.

Revisando a diminuta literatura disponível que discute as questões referentes aos aspectos epide- miológicos e sociais que envolvem a política previdenciária nacional, encontramos Possas $^{2}$, analisando os benefícios concedidos por incapacidade temporária. A autora encontrou os transtornos neuróticos, síndrome de dependência do álcool, outras psicoses não orgânicas e a psicose esquizofrênica como responsáveis, juntos, por cerca de $10 \%$ do total de benefícios concedidos. Quando ainda levou em conta epilepsia ( $1 \%)$, a psicose alcoólica $(0,7 \%)$ e as psicoses afetivas $(0,5 \%)$, esse total se elevou, no ano referido, para $12 \%$ dos benefícios considerados. Outro autor, Narciso3, ao avaliar 1028 segurados em

\footnotetext{
${ }^{1}$ Professor da Disciplina de Neurologia da Universidade Federal de Santa Catarina (UFSC); ${ }^{2}$ Residente de Clínica Médica do Hospital Universitário UFSC; ${ }^{3}$ Médico Epidemiologista do Centro de Estudos do Hospital Universitário UFSC e supervisor médico pericial do INSS de Florianópolis/SC; ${ }^{4}$ Acadêmico de Medicina da UFSC.
}

Recebido 14 Dezembro 1999, recebido na forma final 6 Dezembro 2000. Aceito 15 Dezembro 2000.

Dr. Paulo Cesar Trevisol-Bittencourt - Centro de Estudos do Hospital Universitário/UFSC - 88040-970 Florianópolis SC - Brasil. E-mail: pcb@hu.ufsc.br 
benefícios por patologias diversas, encontrou neuropatias em 71 pacientes, um dado que per si já sugere melhor investigação deste tema.

Por outro lado, de acordo com dados da DATAPREV, no ano de 1996 realizaram-se 13.121 exames médico periciais em neurologia $(4,17 \%$ do total de exames médico periciais) o que gerou um gasto de $\mathrm{R} \$$ 97.580,00 para a Previdência Social. Em psiquiatria realizaram-se 38.758 exames médico-periciais naquele ano (12,33\% do total) com um gasto envolvido de $\mathrm{R} \$ 271.313,00$.

Esperamos, com nosso estudo, despertar o interesse de outros colegas para a realização de estudos similares em outras regiões deste país.

\section{MÉTODO}

Este estudo consiste na revisão dos encaminhamentos realizados pela perícia médica do INSS/Florianópolis/ SC para avaliação neurológica especializada, no período compreendido entre outubro de 1997 e maio de 1998. Foram examinados todos os pacientes encaminhados pela perícia médica do INSS de Florianópolis/SC para um parecer de médico especialista credenciado. As avaliações tinham por objetivo definir diagnóstico e deliberar sobre a capacidade laborativa destes pacientes. O total de 108 pacientes estava em benefício por tempo variável, por apresentar doença neurológica previamente documentada ou por suspeita de algum provável distúrbio neurológico ainda incógnito. Todos os pacientes foram avaliados por um dos autores (PCTB), sendo submetidos a judiciosa anamnese e minucioso exame clínico geral e neurológico. Quando um diagnóstico clínico não era estabelecido após o primeiro contato, outras avaliações eram implementadas. Nestas, a presença de familiares ou testemunhas de eventos era sempre requisitada, para uma complementação das informações prestadas inicialmente pelos pacientes. Muitos deles já vinham ao consultório com exames complementares tais como: tomografia computadorizada, eletroencefalograma, eletromiograma e ressonância magnética. Todos os dados obtidos foram considerados para fins diagnósticos, particularmente se os achados complementares exibiam ou não congruência com os sintomas. Igualmente as informações contidas nos prontuários da Previdência Social também foram levadas em conta e confrontadas com aquelas obtidas através de contato pessoal. Alguns poucos prontuários, por estarem no interior do Estado e pela lenta burocracia, não puderam ser acessados. Igualmente, algumas outras informações, não relevantes para os propósitos deste estudo, foram de difícil obtenção. Devido a estes percalços, nesta pesquisa clínicoepidemiológica limitamo-nos a apresentar os diagnósticos finais de tais pacientes.

Como o parecer do médico-perito especialista em neurologia é apenas um entre os vários fatores que a Previdência Social utiliza para decidir sobre o grau de incapacidade laborativa do indivíduo, resolvemos relacionar as hi- póteses diagnósticas como aptos para o trabalho (sem nenhuma incapacidade), pacientes com incapacidade temporária e/ou relativa (nestes sugeriu-se pagamento de auxílio-doença), pacientes com incapacidade permanente (sugerindo-se nestes aposentadoria por invalidez) e por último, pacientes com capacidade de trabalho indefinida (quando o parecer neurológico isolado não foi suficiente para se chegar a uma conclusão). Foram considerados arbitrariamente na "síndrome pós-TCE", todos aqueles pacientes com distúrbios neurológicos motores ou cognitivos, não epilépticos e com sintomas diversos explicados e provocados primariamente por traumatismo cranioencefálico (TCE). Dentro de "distúrbios reumatológicos", estão incluídos os seguintes diagnósticos: lombalgias inespecíficas, hérnias discais, artrose, sintomas pós trauma com dor crônica sendo o elemento dominante. Para o diagnóstico de epilepsia e julgamento da sua severidade, foi considerada somente a história clínica. Em tais casos, capacidade para o trabalho era arbitrada em função da gravidade da síndrome epilética, confrontada com o tipo de trabalho exercido.

\section{RESULTADOS}

Na Tabela 1 apresentamos as hipóteses diagnósticas dos pacientes submetidos à perícia neurológica, correlacionando-as com seus respectivos graus de incapacidade.

Setenta e nove $(73,15 \%)$ dos pacientes eram homens contra vinte e nove $(26,85 \%)$ de mulheres, totalizando 108 pacientes. A maioria da amostra tinha aproximadamente dois anos de benefício previdenciário.

\section{DISCUSSÃO}

No Brasil, 1 a $2 \%$ da população geral são acometidas por alguma forma de epilepsia ${ }^{4}$. Em nossa amostra, pela Tabela 1, vemos que 35 dos pacientes encaminhados à perícia neurológica para avaliação laborativa tinham epilepsia, sendo este o diagnóstico mais frequente. Em cinco pacientes deste grupo, TCE foi identificado como a etiologia. Em dois outros havia epilepsia secundária a esclerose mesial temporal, ambos com indicação de avaliação cirúrgica ${ }^{5,6}$ e um paciente apresentava o diagnóstico de neurocisticercose (NC). Possas ${ }^{2}$ relata a epilepsia como responsável por $1 \%$ dos benefícios concedidos por incapacidade temporária no Brasil em 1986. Narciso ${ }^{3}$ encontrou epilepsia em $1,46 \%$ dos pacientes numa amostra de 1028 segurados em benefício.

Em nossa pesquisa, esperavamos previamente que epilepsia fosse o carro chefe e isso realmente ocorreu. Entretanto, quanto à etiologia, a baixa incidência de NC foi surpreendente, pois estudos recentemente publicados ${ }^{4,7}$ revelaram ser esta condi- 
Tabela 1. Diagnósticos e grau de incapacidade laborativa em pacientes encaminhados à perícia neurológica realizada pelo INSS de Florianópolis/SC, de outubro de 1997 a maio de 1998.

\begin{tabular}{|c|c|c|c|c|c|c|}
\hline Diagnósticos & $\mathrm{N}^{\circ}$ de pacientes & $\%$ & $\begin{array}{c}\text { Pacientes } \\
\text { aptos para o } \\
\text { trabalho }\end{array}$ & $\begin{array}{c}\text { Pacientes com } \\
\text { incapacidade } \\
\text { temporária }\end{array}$ & $\begin{array}{l}\text { Incapacidade } \\
\text { permanente }\end{array}$ & $\begin{array}{c}\text { Capacidade } \\
\text { de trabalho } \\
\text { indefinida }\end{array}$ \\
\hline Epilepsia & 35 & 32,40 & 4 & 15 & 6 & 10 \\
\hline Distúrbios Reumatológicos & 18 & 16,66 & - & 4 & 6 & 8 \\
\hline Distúrbios Psiquiátricos: & 16 & 14,81 & 2 & 5 & - & 9 \\
\hline 1-Dist. Conversivo/ simulação & 8 & - & 1 & 1 & - & 6 \\
\hline 2 - Depressão & 5 & - & - & 3 & - & 2 \\
\hline 3 - Outros & 3 & - & 1 & 1 & - & 1 \\
\hline Polineuropatia: & 10 & 9,25 & 1 & 2 & 5 & 2 \\
\hline 1-Alcoólica-desnutricional & 7 & - & 1 & 1 & 3 & 2 \\
\hline 2 - Outras & 3 & - & - & 1 & 2 & - \\
\hline Síndrome pós-TCE & 8 & 7,40 & - & 2 & 2 & 4 \\
\hline Doença Cérebro-Vascular & 7 & 6,48 & - & 4 & 3 & - \\
\hline Cefaléia & 3 & 2,77 & 1 & 1 & - & 1 \\
\hline Diagnóstico a esclarecer & 7 & 2,77 & - & 4 & 3 & - \\
\hline Síndrome Tunel do carpo & 2 & 1,85 & - & 1 & - & 1 \\
\hline Parkinson & 1 & 0,92 & - & 1 & - & - \\
\hline Neoplasia Cerebral & 1 & 0,92 & - & - & - & 1 \\
\hline Síndrome vestibular periférica & 1 & 0,92 & - & - & - & 1 \\
\hline Doença de Little & 1 & 0,92 & - & 1 & - & - \\
\hline Doença de Machado-Joseph & 1 & 0,92 & - & - & - & 1 \\
\hline Surdo - Mudez & 1 & 0,92 & - & - & - & 1 \\
\hline Total & 108 & 100 & 8 & 39 & 22 & 39 \\
\hline
\end{tabular}

ção a causa mais comum de epilepsia na nossa sociedade. É possível que este dado reflita um prognóstico distinto das epilepsias secundárias a NC, quando comparadas com outras formas de epilepsias parciais sintomáticas. Em resumo, será que epilepsia por NC entra em remissão mais facilmente que outras? Estudos com delineamento apropriado deverão ser implementados para melhor entendimento desta questão.

Por outro lado, quando analisamos a relação entre epilepsia e capacidade para o trabalho, de acordo com a Tabela 1, veremos que, dos 35 pacientes com epilepsia, em 4 não houve alteração laborativa, mas em 6 pacientes havia incapacidade permanente para o trabalho, mostrando o seu potencial para diminuição de capacidade laborativa. A maioria destes pacientes apresentavam epilepsia parcial, e nestes, dois pontos nos pareceram fundamentais no incremento da incapacidade. Habitualmente são formas de epilepsia resistentes a terapêutica medicamentosa e por esta razão, tais pacientes acabam utilizando doses elevadas de drogas anti-epiléticas (DAE) diversas. Estas, por sua vez, estão implicadas com inúmeros efeitos colaterais, alguns dos quais extremamente limitantes 5 . Desta maneira, não conseguimos discernir com precisão qual destes fatores contri- buiu para a restrição da capacidade laborativa: se a epilepsia, seu tratamento, ou ambos. Este é um detalhe que igualmente necessita ser esclarecido em investigações futuras.

Curiosamente, distúrbios reumatológicos aparecem pela Tabela 1 como a segunda causa de consulta em perícia neurológica. $\mathrm{Narciso}^{3}$ encontrou este grupo diagnóstico como o líder em benefícios pagos pela Previdência Social. Apesar de muitos diagnósticos deste grupo não serem genuínos problemas neurológicos, eles estão aqui provavelmente pela necessidade do perito precisar de um parecer neurológico para embasar sua decisão. Além deste aspecto, é notável que apenas $73 \%$ dos pacientes atendidos em um ambulatório de neurologia sejam reais sofredores de típicos problemas neurológicos ${ }^{8}$.

Durante o período pesquisado, também outros pacientes com transtornos de natureza não neurológica foram encaminhados. Entre estes, o destaque ficou com distúrbios psiquiátricos, representando a nossa terceira causa de consulta em perícia neurológica, perfazendo quase $15 \%$ do total de pacientes (note-se ainda pela tabela 1 que ninguém deste grupo apresentava incapacidade permanente). Tais encaminhamentos de casos psiquiátricos para avali- 
ação neurológica, muito provavelmente ocorrem quando uma base orgânica é suspeitada para supostos sintomas neurológicos e, em tais casos, seria fundamental uma avaliação neurológica especializada. Na metade destes pacientes, foi julgado tratar-se de distúrbio conversivo ou simulação, ficando o diagnóstico diferencial muitas vezes difícil.

As manifestações conversivas são conhecidas e estudadas desde há muito; porém, a dramaticidade de suas características clínicas continua surpreendendo a médicos e leigos. Exemplificando, uma paciente que estava há cerca de 2 anos em benefício por "AVC", por recomendação de colega especialista, apresentava sinais e sintomas "shakesperianos" típicos de hemiplegia histérica. Aliás, nesta área, este diagnóstico assim como crises psicogênicas bizarras, frequentemente confundidas com epilepsia, foram aqueles mais comumente encontrados. Em alusão ao tratamento de distúrbios histéricos, o renomado Dr. Babinski dizia no princípio deste século "...cura-se a histeria por meio de uma ação persuasiva, enérgica e hábil" ${ }^{\prime 9}$. Sinceramente, achamos que princípios fundamentados em autoritarismo, ao invés de a atenuarem, tendem a incrementá-la, sendo imperativa uma orientação especializada adequada em todos estes casos.

A simulação é outro fato que tem grande importância em perícia médica. Aqui observa-se uma forma especial de relacionamento médico-paciente, notoriamente diferente da habitual. $O$ paciente vem em busca não de um diagnóstico ou tratamento, confiante no profissional médico, mas na procura de um benefício de que necessita ou a que julga ter direito. O médico-perito pode ser interpretado como um obstáculo a superar, surgindo daí conflitos na relação paciente-médico. Este, por sua vez, deverá ser justo para não negar o que é legítimo, nem conceder graciosamente o que não é devido. Deve ainda ter alguns atributos de personalidade e caráter, como serenidade para não se deixar envolver por pressões externas ou mesmo fatores extra-doença, problemas sociais, desemprego e outros apelos a que está sujeito ${ }^{10}$. Finalmente, percebe-se a simulação pelo exame neurológico normal, exagero nas queixas fictícias e várias e grotescas tentativas de mimetização de um estado mórbido incompatível com seu exame clínico. Enfim, tal estado poderia ser sumarizado pela combinação de riqueza sintomatológica com extrema pobreza semiológica.

Outros diagnósticos, ainda dentro do grupo dos distúrbios psiquiátricos, foram depressão, ansieda- de e neurose. Portanto não se pode deixar de falar em distúrbios somatoformes na sociedade moderna11,12. Em serviços de neurologia, a somatização pode chegar até a $18 \%$ no sexo feminino ${ }^{13}$. Pacientes com sintomas somatoformes são ávidos consumidores dos recursos da saúde, representando uma importante carga econômica para os serviços de saúde e Previdência Social ${ }^{13}$. É evidente que também contribui neste aspecto a obsessão médica em avaliar compulsiva e exaustivamente estes pacientes, com o intuito de "descobrir sua causa". É interessante citar que estes pacientes comumente vinham para a avaliação repletos de exames complementares sofisticados, onerosos e que, no decorrer do exame clínico, se revelavam inúteis.

Alcoolismo, por sua vez, foi o diagnóstico principal em 7 de nossos pacientes e em 3 destes levou a incapacidade definitiva. De acordo com Kurtzke', nos EUA a incidência anual de alcoolismo é de 10 por 100.000 habitantes e prevalência anual de 500 por 100.000, já considerando apenas os alcoolistas que necessitem de um parecer neurológico. Caso contrário os índices seriam muito maiores, pois, de acordo com aquele autor, somente $10 \%$ dos alcoolistas, necessitam de diagnóstico ou tratamento por neurologista. De acordo com o Programa Nacional de Controle dos Problemas Relacionados com o Consumo de Álcool - PRONAL ${ }^{14}$ a prevalência da síndrome de dependência de álcool mais o abuso do álcool (ou ingestão patológica) têm sido estimados em torno de 5 a $10 \%$ da população adulta o que compreenderia 3,5 a 7 milhões de pessoas. E ainda $40 \%$ das consultas prestadas pelo ministério da Previdência Social foram para pacientes com abuso de álcool, sendo o alcoolismo isoladamente a $8^{\circ}$ causa de requerimento de concessão de auxílio-doença. Outra forma de polineuropatia encontrada foi aquela associada ao diabetes mellitus, diagnóstico principal em dois pacientes da casuística ${ }^{14}$.

Um fato marcante na avaliação neurológica de diversos pacientes foi TCE. Além de incidir em 5 pacientes do grupo da epilepsia, também causou sequelas distintas em mais de 8 pacientes (Síndrome pós-TCE). Ilustra-se que TCE e sintomas relacionados, foram identificados como a quarta causa de consulta em ambulatório de neurologia do $\mathrm{HC}$ da FMUSP, com 3,93\% dos pacientes ${ }^{8}$.

Gostaríamos de ressaltar os aspectos neuropsicológicos dos traumas cranianos ${ }^{15}$, pois a frequência e eventual gravidade do comprometimento cognitivo que se segue aos TCEs parecem ser desconhecidas 
da população leiga e lamentavelmente também dos médicos, o que justificou a expressão "epidemia silenciosa" por Parker ${ }^{15}$. Achados neurológicos normais frequentemente levam a conclusão errônea de que os sintomas pós-injúria, cognitivos e emocionais, são de natureza psicológica. Os distúrbios comportamentais secundários a lesão axonal difusa (LAD) parecem ser ignorados pela maioria dos neurologistas. A LAD é considerada o mecanismo fisiopatológico básico dos traumatismos cranianos leves e também participa em outros de maior gravidade, sendo a responsável pelas alterações neuropsicológicas encontradas nestes pacientes (déficits cognitivos variados, distúrbios de memória e de atenção, alterações de personalidade, do humor e até psicose), contribuindo de forma significativa para a incapacitação laborativa destes indivíduos. A incidência anual de distúrbios neuropsicológicos persistentes é de 370 por 100.000 , portanto muito maior que a incidência de esquizofrenia ${ }^{15}$. Vale lembrar que casos graves de LAD podem ser observados na ausência de sinais neurológicos focais ou anormalidades na tomografia ou ao EEG ; aliás, é geralmente não detectada nestes exames. Ressonância magnética é o único exame atual que poderá fornecer indícios sugestivos.

Já quando enfocamos as doenças vasculares cerebrais, verificamos, mais do que em qualquer outro grupo diagnóstico, a importância de outras entidades clínicas de base como é o caso de hipertensão arterial sistêmica (HAS), sempre presente naqueles pacientes encaminhados com esta suspeição diagnóstica. Dada a alta incidência de HAS e suas complicações na população em geral, tínhamos a expectativa prévia de encontrar maior percentual destes casos. Contudo, é bastante provável que a fácil identificação da sequela neurológica e sua respectiva incapacidade tornem dispensável um parecer do perito especialista em neurologia. Pensamos ser esta a justificativa mais racional para o número inexpressivo de tais casos em nossa amostragem.

Em resumo, dos 108 pacientes encaminhados para parecer neurológico sobre sua capacidade de trabalho: em 22 havia incapacidade permanente (sugerindo-se aposentadoria por invalidez); 39 apresentavam incapacidade parcial (recebendo auxílio doença); os restantes foram considerados aptos.

Finalmente, gostaríamos de reafirmar que, se por um lado a doença neurológica pode ter um potencial destrutivo sobre a capacidade laborativa do indivíduo, por outro lado o potencial de reabilitação des- tes pacientes é grande e frequentemente subestimado, até por especialistas ${ }^{9}$. Dizemos isto, principalmente por dois motivos: primeiro, porque algumas pesquisas prévias ${ }^{16}$ mostram que a incapacidade prolongada outorgada de forma inadequada pode chegar a $41,3 \%$; segundo, porque atualmente muito se tem feito pela sobrevida do paciente neurológico; todavia, muito pouco tem sido feito para sua efetiva reabilitação. Em parte, esta distorção é devida a descrença sobre as potencialidades de restauração orgânica e recuperação funcional do incapacitado; porém, não devemos minimizar a participação do massacrante ritual burocrático durante o período de reabilitação. Devido a isso, muitas vezes, não restará outra opção para o médico perito além da aposentadoria prematura por invalidez.

Agradecimentos - Adriana Eich foi fundamental para transformar uma idéia em artigo científico. No decurso deste trabalho um dos autores (MAF) perdeu um ente querido, mais uma vítima de TCE por acidente automobilístico. Este estudo é dedicado a este nobre cidadão. Os funcionários do setor de Perícias Médicas do INSS de Florianópolis/SC, foram pessoas educadas e essenciais na obtenção dos dados, por isso nossa gratidão é extensiva a todas elas.

\section{REFERÊNCIAS}

1. Kurtzke JF. The current neurologic burden of ilness and injury in the United States. Neurology 1982;32:1207-1214

2. Possas C. A especificidade do quadro sanitário no Brasil: principais características. In Possas A (ed). Epidemiologia e Sociedade: heterogeneidade estrutural e saúde no Brasil. São Paulo: HUCITEC; 1989:19-115.

3. Narciso HR. Perícia médica em Blumenau. Arq Cat Med 1991;20:37-38.

4. Rigatti M, Trevisol-Bittencourt PC. Causas de epilepsia tardia em uma clínica de epilepsia do Estado de Santa Catarina. Arq Neuropsiquiatr 1999;57:787-792.

5. Sander JWAS, Duncas JS, Trevisol-Bittencourt PC. O tratamento das crises epiléticas. Arq Cat Med 1996;15:77-84.

6. Trevisol-Bittencourt PC. Alguns comentários sobre epilepsia do lobo temporal. Arq Cat Med 1990;19:45-48.

7. Trevisol-Bittencourt PC, Silva NC, Figueredo R. Neurocisticercose em pacientes internados por epilepsia no Hospital Regional de Chapecó Região Oeste do Estado de Santa Catarina. Arq Neuropsiquiatr 1998;56:53-58.

8. Ferri-de-Barros JE, Nitrini R. Que pacientes atende um neurologista? Arq Neuropsiquiatr 1996, 54:637-643.

9. Leitão A. Reabilitação neurológica. Rio de Janeiro: Arte Nova, 1972.

10. Manual do médico-perito. 3.ed. Brasília: MPS, INSS, Coordenação Geral de Serviços Previdenciários, 1993.

11. Estañol B. Invalidez em padecimentos neurológicos. Simposio la Invalidez sus causas y su repercusión socioeconómica. Gazeta Med Méx 1982:118:171-185.

12. Lopez TR, Haces MC. Incapacidad y limitaciones de la actividad laboral en pacientes neuróticos. Rev Hosp Psiquiatr Habana 1994;35:127-130.

13. Martinez DMC. Costo de los sintomas somatoformes. Rev Med IMSS (Méx) 1997;35:31-36.

14. Ramos, SP. Bertolote JM. Problemas sociais relacionados ao consumo de álcool. In: Alcoolismo hoje. 3.Ed. Porto Alegre: Artes Médicas; 1997:131-138

15. Mattos P. Aspectos neuropsicológicos dos traumatismos cranianos. J Bras Psiquiatria 1992;41:495-502.

16. Islas GM. Incapacidades prolongadas inadecuadas, un problema del assegurado, del medico o de los servicios de salud? Rev Med IMSS (Méx) 1996:34:379-384 